\title{
MOOC Teaching Mode of News Transmission Based on Network Audio Data Decoding Technology
}

\author{
https://doi.org/10.3991/ijet.v13i06.8582 \\ Chune Shen \\ Hubei University of Arts and Science, Xiangyang, China \\ 564842959 @qq. com
}

\begin{abstract}
MOOC, an online classroom development mode, has gradually developed due to its unique advantages under the background of big data. However, most online audio data of MOOC are acquired through the application software on PCs, which has limitations in the support of audio format. In the meanwhile, there are still some shortcomings in the existing research on the application and integration of the core technology of MOOC. Therefore, this study constructs the News Transmission MOOC course based on the network audio data decoding technology. Moreover, the course is applied to the actual teaching process to test the teaching effect and realize the organic integration of the core technology of computer and the teaching idea of MOOC. The practical teaching results show that the MOOC course not only improves the teaching effect, but also has significance and value for improving students' ability and learning interest.
\end{abstract}

Keywords-Network audio data decoding technology; MOOC; multimedia; big data

\section{Introduction}

The third revolution of science and technology in western countries, namely, the revolution of information technology, has swept the world for several decades, exerting profound influence on various industries. As the information technology revolution develops to a new stage, artificial intelligence, cloud computing and big data have made great breakthroughs, which have greatly changed our life, work and learning styles [1]. Particularly, the frontier information technology represented by big data has been extensively used in all walks of life due to its excellent data analysis and data mining capabilities. MOOC is the abbreviation of Massive Open Online Course. MOOC [2] refers to a teaching and learning approach which rapidly grows with the fast development of computer technology and Internet technology with the characteristics of openness and online. Course providers publish the courses on Internet server and thus learners can access the course resources through the Internet at anytime and anywhere to complete the study. In other words, the learners can break through the limits of time and space to learn courses based on MOOC. Due to the advantages of spanning time and space, MOOC has made great progress in recent years. At present, 
numerous famous universities at home and abroad begin to publish MOOC courses on the Internet, covering mathematics, computer science and other natural sciences [3], as well as multiple disciplines such as engineering, social science and humanities [4].

Instructors' audio data which is the core content of MOOC, are continuously transmitted to learners in the progress of MOOC course. The audio information generated by instructors is analog signal, while the computer and the Internet transmit digital signal. Therefore, it is necessary to sample and code audio information using technical means before it is published in MOOC course. Learners' client must decode the information into audio analog signal and then obtain the contents published by instructors. To guarantee that the audio information of instructors can be timely and accurately transmitted to learners, the network audio data decoding technology contained in MOOC should be both real-time and correct. Due to the limitation of time and space, the traditional teaching form can hardly meet the learning needs of various groups. Therefore, MOOC with high freedom degree can break the restrictions of time and space, which has become the inevitable choice of news transmission related courses.

\section{State of the art}

With the development of mobile Internet and the popularity of smart phones, the way of news transmission has changed dramatically. The rapid development of social media and internet media has altered the news transmission way dominated by the traditional media such as TV and newspapers. In the meanwhile, the emerging selfmedia, as an important form of newsTransmission, has great advantages over transmission speed and transmission scope compared with the traditional media. Under the dramatical changes, the teaching methods of journalism and language related majors can hardly meet the requirements of the news transmission industry. At the same time, the traditional multimedia teaching method has some defects in participation and interaction, requiring changes to adapt to the changes. Therefore, domestic and foreign scholars have carried out extensive and in-depth research on the teaching of journalism and language majors. Barak [5] applied MOOC teaching to English and Arabic language courses, concluding that both the intrinsic motivation and learning autonomy of MOOC participants are improved. Zhuhadar et al [6] envisioned a new generation of MOOCs which support formal semantics interpretability through online social networks and SemanticWeb. Compared with the existing platforms of MOOCs, semantic technology supports more flexible management of information. The experimental results demonstrate that MOOCs teaching is conductive to promoting students' understanding and learning. Recently, a large number of open online courses have emerged in Spain, which have been proved to be an effective way of foreign language teaching and learning. The course conducted by Chacón-Beltrán [7] is an experience in a large student group with MOOC as a learning tool of Spanish English. The experience course aims to enable learners to quickly learn the meaning of the most common 1,000 words in English and begin to read short sentences. According to the experimental results, the teaching effects of MOOC have been recognized by both 
teachers and students. In China, Li Liang et al. [8] deeply discussed the problems existing in the teaching of news transmission under the trend of media convergence as well as the improvement strategies. Besides, they believed that the optimization of curriculum system and the integration of teaching mode should be the core contents of teaching improvement. A research team [9] focused on the teaching reform strategy of writing, the core course in the teaching of journalism and transmission, and deeply studied the theoretical basis and advantages of introducing audiovisual resources into the curriculum to assist teaching. Briefly, MOOC has realized the scale and globalization of the audience of high-level university teaching resources through the standardized online course teaching, expanding the knowledge imparting chain of the traditional higher education [10]. However, there still remain some problems in teaching practice and technology implementation of MOOC. Firstly, in most cases, MOOC acquires network audio data based on application software on PCs, which has limitations on the support of audio format [11]. Secondly, the existing research still has some shortcomings in the application and integration of the core technology, which only focuses on the construction of curriculum framework. The integration of core technology, especially the decoding of network audio data, is of significant importance to curriculum construction. It undertakes the task of accurately and timely transferring the course resources provided by instructors to learners. Moreover, compared with the traditional open distance education system, MOOC is only at the level of course, which lacks digital teaching resource database as well as data exchange and sharing with other teaching and management platforms. This is far from the requirements of UNESCO for the standards of open education resources.

The innovation of this study is mainly reflected in the following two perspective: Firstly, MOOC is organically combined with News Transmission course to construct a complete computer teaching mode of News Transmission, thus providing a highly efficient teaching environment for the course development. Secondly, network audio data decoding technology is integrated into the construction of the teaching mode of MOOC, which offers a solid technical guarantee for the timely and accurate transfer of course resources to learners. Besides, the implementation of MMS protocol on the embedded ARM platform is completed. MMS protocol is a private streaming media protocol of Microsoft, and its specification is confidential. In addition, some open source software deduces the content of MMS protocol by fetching the received and sent packets when MMS streaming media data is played by WMP (Windows Media Player). According to the specific content of MMS protocol, the MMS function is successfully realized on ARM platform using $C$ language. The computer teaching model based on the teaching environment of MOOC constructed in this study provides a new teaching mode for the corresponding teaching. 


\section{Theoretical construction}

\subsection{Application of motivation theory in MOOC}

This study mainly introduces motivation theory to MOOC. Motivation theory [12] holds that the learning process can truly achieve the desired effect only if the learners fully possess the learning motivation and develop the meaning of learning knowledge. This can effectively stimulate learners' creative consciousness in the learning process, which further improves the learning effect. In the teaching environment of MOOC, learners can independently control the learning process according to their needs and learning conditions and can also make use of the functions provided by the teaching model so as to communicate with other learners on the course content in real time, thus strengthening the learning effect. Figure 1 shows the structure of motivation learning theory.

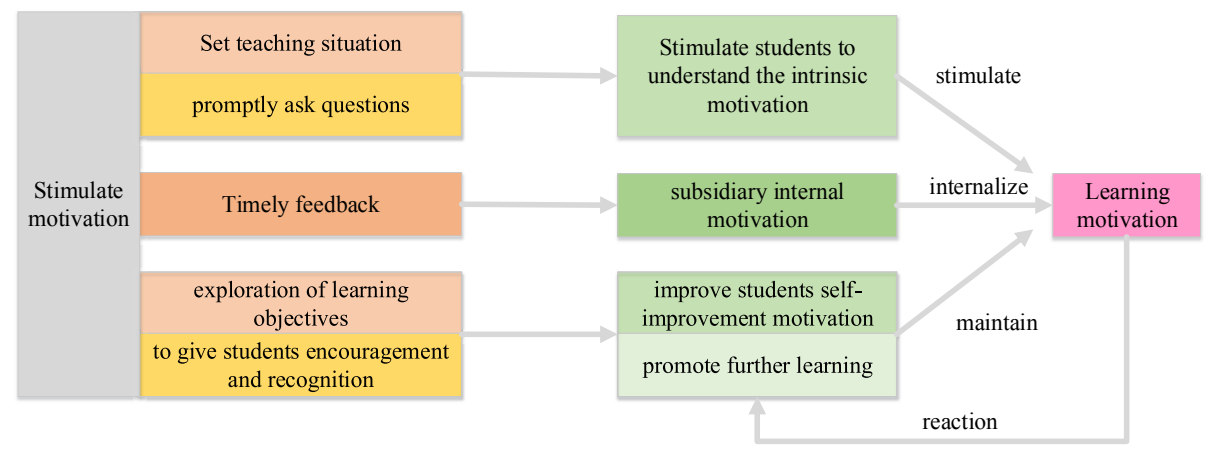

Fig. 1. Structure of motivation learning theory

\subsection{Application of MMS Protocol in MOOC}

MMS is a private streaming protocol of Microsoft, which transmits a series of realtime multimedia broadcasts, videos, audio tracks and live data on the internet. The users can receive real-time video or audio through a computer or a dedicated receiving terminal. The media files on the MMS multimedia server exist in the form of ASF (Advanced Streaming format) or WMA. Besides, MMS protocol sends relevant data to users from multimedia server to their computers in the form of data packets and data blocks. The client-side flow chart of MMS is presented in Figure 2.

\subsection{Application of network audio data decoding technology in MOOC}

Current; $\mathrm{u}$, most audio data on the Internet are stored and transmitted in MP3 audio format characterized by high compressibility and low distortion. In the meanwhile, it can adjust the bit rate according to the bandwidth of the transmission channel. Consequemtly, it is suitable for audio data transmission in computer network environment. 
After the Huffman encoding data and the relevant coding parameters are read from the MP3 data frame, the audio data decoding process can be carried out to output the audio signal. The decoding of audio data consists of three processes, respectively, inverse quantization of Huffman coding data, inverse cosine transform, windowing operation as well as frequency inversion and synthesis multi-term filtering. The network audio data decoding process is shown in Figure 3.

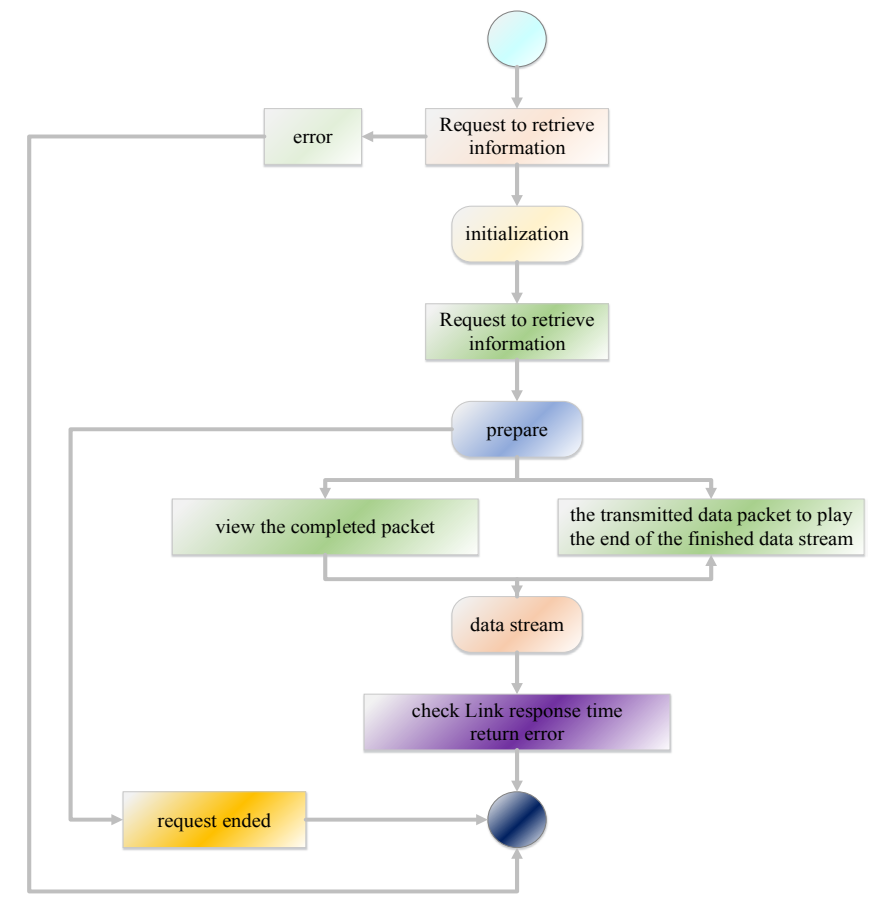

Fig. 2. Client-side flow chart of MMS

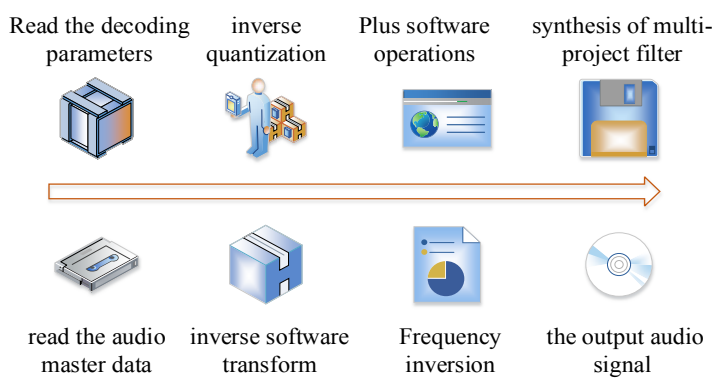

Fig. 3. Network audio data decoding process

Inverse quantization. $x_{i}$ is the received Huffman coding data. $y_{i}$ is the results obtained by inverse quantization. Inverse quantization is achieved using formula (1) formula (3): 


$$
\begin{gathered}
y_{i}=\operatorname{sign}\left(x_{i}\right) \operatorname{abs}\left(x_{i}\right)^{(4 / 3)} 2^{u 1} / 2^{u 2} \\
\mathrm{u} 1=\frac{1}{4}\left(\text { global }_{\text {gain }[g r][c h]}-210\right)
\end{gathered}
$$

$\mathrm{u} 2=$ scalefac_multiplier-(scalefac 1 [gr] $[\mathrm{ch}][\mathrm{sfb}]+\operatorname{preflag}[\mathrm{gr}][\mathrm{ch}]$ pretab[sfb]

Inverse cosine transform. After completing the inverse quantization of Huffman coding data, the obtained data is conducted with inverse cosine transform, and the transform formula is shown in formula (4):

$$
\mathrm{X}_{i}=\sum_{k=0}^{\frac{n}{2}-1} X_{k} \cos \left(\frac{\pi}{2 n}\left(2 i+1+\frac{n}{2}\right)(2 k+1) \ldots i=0,1,2, \ldots, n-1\right.
$$

Windowing Operation. After the inverse cosine transform, the output results need to be windowed in accordance with different window types of MP3 data frames. Different window functions are required for various types of blocks. The block type parameters exist in the header of the MP3 data frame. Based on the block types, the window function is shown as below:

When the block type is 0 (normal frame):

$$
\mathrm{Z}_{\mathrm{i}}=\mathrm{x}_{\mathrm{i}} \sin \left(\frac{\pi}{36}\left(i+\frac{1}{2}\right)\right) \ldots . .0 \leq i<35
$$

When the block type is 1 (start frame):

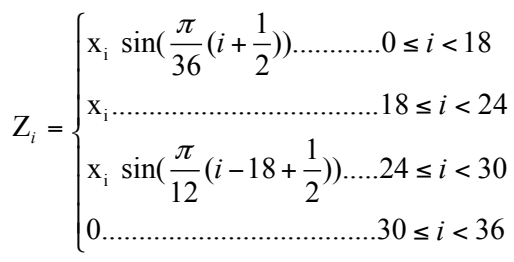

When the block type is 3 (end frame):

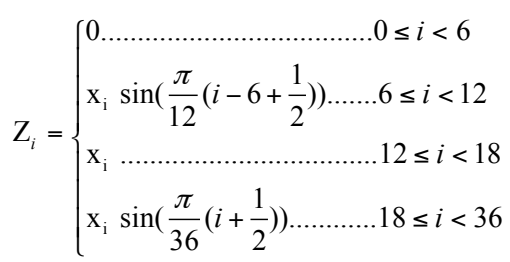

Frequency inversion and synthesis multi-term filtering. After conducting the windowing operation, the obtained data are processed with frequency inversion and synthesis multi-term filtering. Frequency inversion aims to compensate coding and improve the efficiency of cosine transform. The synthesis multi-term filtering synthesizes the data obtained after frequency inversion and outputs the final audio signal. 


\section{$4 \quad$ MOOC of News Transmission based on network audio data decoding technology}

\subsection{Teaching example}

Construction of teaching mode. In this study, MOOC course of News Transmission based on network audio data decoding technology is constructed through modularization. That is to say, the realization of MOOC is divided into different modules according to the functions. After the implementation of all functional modules, they can be integrated into a complete course by linking each other. This construction method mainly has two advantages. Firstly, by dividing a large and complex system into smaller and easier functional modules, the whole system is realized through the integration of modules, which can effectively reduce the difficulty of implementation and also improve the platform building efficiency and robustness. Secondly, when system operation occurs problems, the modular construction method can quickly locate the specific module and implement the corresponding repair scheme, thus effectively improving the stability of the system.

The whole teaching mode construction process includes three steps including decomposition and definition of modules, realization of modules, and integration and construction of MOOC courses. MOOC is a new educational technology based on the development of computer technology and Internet technology. The main form is that the course provider publishes the course content in the network server. Then, learners can obtain the data of the course content through the Internet and present the complete curriculum environment after processing the local program. On the whole, MOOC course is constructed on the basis of server-client structure. At the same time, it can provide the statistical data generated by learners during the learning process to the course providers so as to improve the course content, which is a main characteristic of MOOC. Therefore, the corresponding modules should be provided in the construction of MOOC course to support this property.

Based on the above analysis, News Transmisson MOOC course based on the decoding technology of network audio data mainly includes the following parts: (1) MOOC course content server: this component is used to store all the course resources needed in the course and upload the course resources produced by the course provider to the content server. (2) Data statistic device: as one of the core components, this module provides the corresponding support to the collection and analysis of the teaching and learning data in MOOC course. (3) Network router: this module is a network component connecting the background module and the foreground module, exerting the function of data transmission. (4) Data access component: this module receives the course data of the MOOC course server from the network router and reversely sends learners' statistic data in learning. (5) Teaching client-side: the module is a client-side program for learners to receive MOOC course. (6) Data storage device: the module temporarily stores the course resource data sent by the course server in order to avoid the impact of network fluctuation on learning. (7) Teaching peripheral equipment: this equipment is set to assist students in their learning process. (8) Net- 
work audio data decoding component: this module decodes the network audio data transmitted by the MOOC course server to convert it into audio signals.

The MOOC course content server adopts the structure of Windows platform and MySQL database is used to support the persistence layer in the course content data storage. In the application layer of course content server, Java programming language is adopted to realize the interface of course content acquisition. Client program can obtain course content data from the server through this interface. Data statistic device module and data storage device mainly store students' learning data and course content data obtained by the client. This study adopts MySQL database to store the data. Besides, Java programming language is used in data access module and teaching client module. Data transmission among these modules is implemented using Socket network data transmission mode.

In the current work, network audio data decoding technology is one of the core components of MOOC course constructed. The basic decoding principle and flow have been explained in the section of theoretical basis. In this study, the objectoriented programming technology is used to implement the network audio data decoding tool class, which realizes the concrete function of each link in the decoding process and the decoding of network audio data.

After the realization of each specific module, these modules are integrated through a certain structure to form a complete MOOC course platform. To be specific, the MOOC course content server is connected to the data statistics device, and the data statistics device is linked to the network router. Besides the other end of the network router is connected to the data access component of MOOC client program. The data access component is connected with the network audio data decoding component. Then, the audio data is decoded and transferred to the MOOC client. To provide the functions of data storage and auxiliary function service, data storage devices and teaching peripheral equipment are connected to teaching client. The structure of each module in the system is shown in Figure 4.

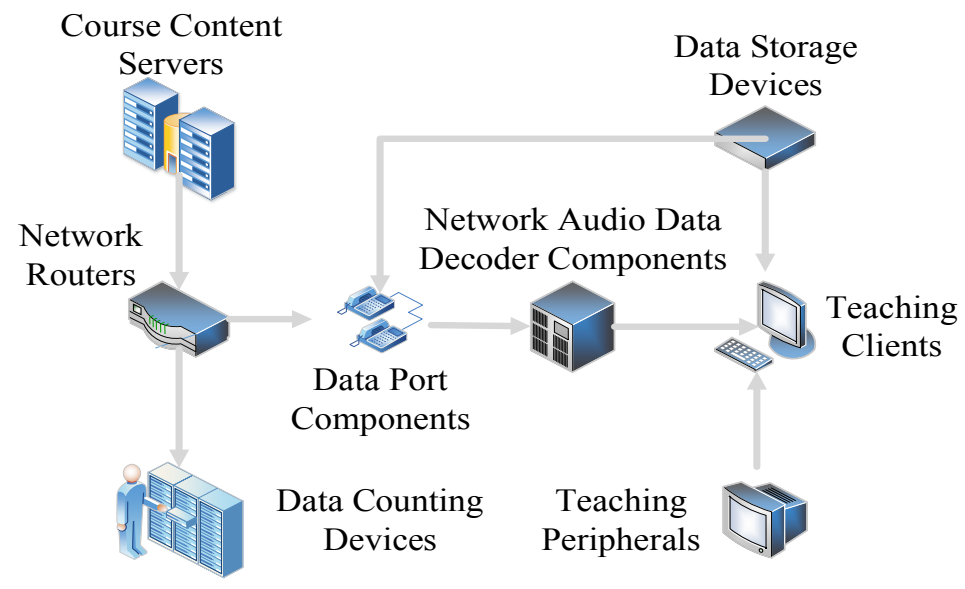

Fig. 4. Structure of News Transmission MOOC course based on network audio data decoding technology 
Practice of teaching mode. Figure 4 and Figure 5 show the construction results of News Transmission MOOC course based on network audio data decoding technology. Figure 5 presents the login interface of MOOC course. Learners can enter the course system by entering the user name and login password. Figure 6 shows the tablet use step of MOOC course. In this interface, learners can not only study the relevant course resources, but also communicate with other learners. In the meanwhile, compared with the traditional teaching methods, learners can control their learning schedule according to their time arrangement. After they log off the system, the previous learning schedule will be automatically connected in next login,

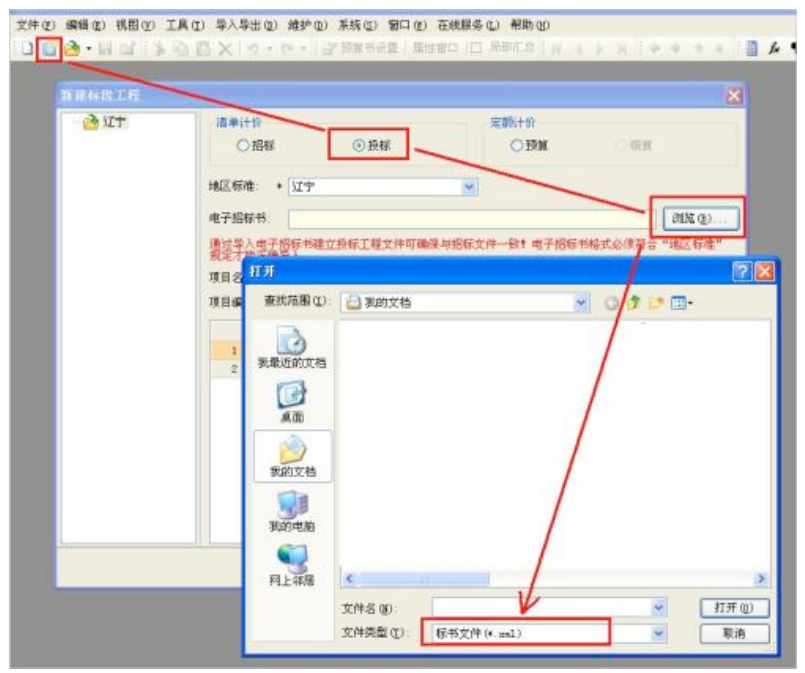

Fig. 5. Login interface I of News Transmission MOOC course based on network audio data decoding technology

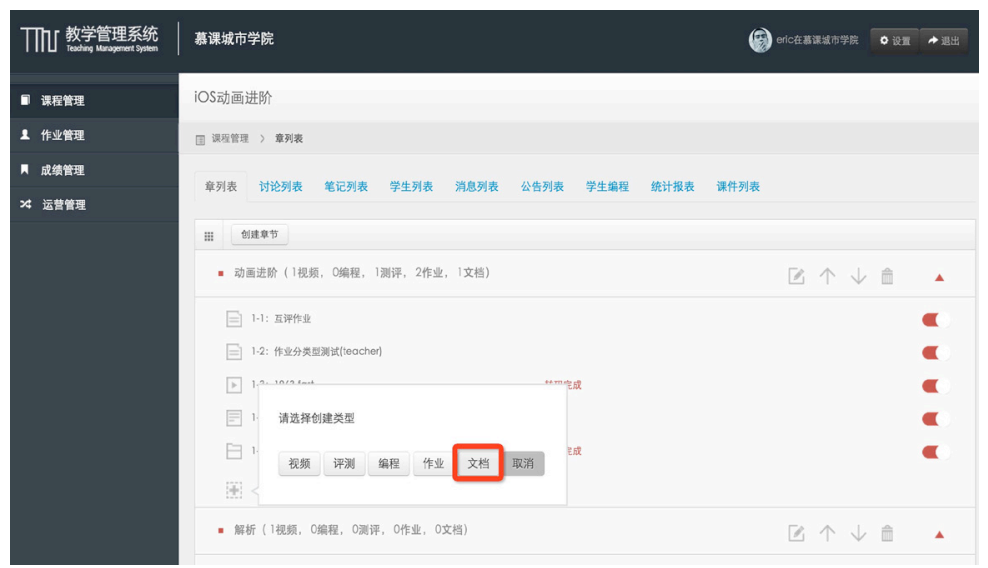

Fig. 6. Login interface II of News Transmission MOOC course based on network audio data decoding technology 


\subsection{Teaching effect}

In order to test the teaching effects of MOOC course constructed in this paper, the course was given to 90 undergraduate in two classes of journalism major in 2016.

The 45 students in one class were taught through the traditional teaching method as a control class. The 45 students in the other class received MOOC course in the learning of News Transmission. To minimize the uncertainty caused by the other factors, the course contents were arranged and taught by the same teacher and corresponding test questions were designed to test the learning effect of the two classes of students.

The test questions are the same for both groups, and the test scores are listed in Table 1. Obviously, the score distribution of the class receiving the Moo course is better than that of the class using the traditional teaching.

In addition to testing the teaching effect using test paper, a survey on the teaching satisfaction of MOOC course among the students was carried out through questionnaire. The questionnaire includes 6 questions, each of which contains three dimensions of approval, general and disapproval. The results of the questionnaire survey are shown in Table 2. Most students believe that MOOC course can effectively improve their autonomous learning ability, self-management ability, Transmission skills and teamwork ability. Additionally, MOOC course has also been recognized by most students in terms of stimulating learning interest and enhancing the knowledge mastery degree.

Table 1. Comparison of test results between the traditional teaching class and MOOC teaching class

\begin{tabular}{|l|c|c|c|c|c|c|}
\hline \multicolumn{1}{|c|}{ Group } & Average scores & $\mathbf{<} \mathbf{6 0}$ & $\mathbf{6 0 - 6 9}$ & $\mathbf{7 0 - 7 9}$ & $\mathbf{8 0 - 8 9}$ & $\mathbf{\geq 9 0}$ \\
\hline Control group $(\mathrm{n}=45)$ & 75.4 & 7 & 14 & 12 & 9 & 3 \\
\hline Experimental group $(\mathrm{n}=45)$ & 81.5 & 2 & 4 & 15 & 16 & 8 \\
\hline
\end{tabular}

Table 2. Investigation results on the teaching satisfaction of MOOC course

\begin{tabular}{|l|c|c|c|}
\hline \multicolumn{1}{|c|}{ Questions } & Agree & Normal & Disagree \\
\hline Improving autonomous learning ability & $41(91.11 \%)$ & $3(6.67 \%)$ & $1(2.22 \%)$ \\
\hline Improving self-management ability & $39(86.67 \%)$ & $3(6.67 \%)$ & $3(6.67 \%)$ \\
\hline Develop Transmission skills & $42(93.33 \%)$ & $1(2.22 \%)$ & $2(4.44 \%)$ \\
\hline Developing teamwork ability & $40(88.89 \%)$ & $3(6.67 \%)$ & $2(4.44 \%)$ \\
\hline Stimulating learning interest & $38(84.44 \%)$ & $2(4.44 \%)$ & $5(11.11 \%)$ \\
\hline Enhancing the mastery degree of knowledge & $39(86.67 \%)$ & $4(8.89 \%)$ & $2(4.44 \%)$ \\
\hline
\end{tabular}

It can be seen that News Transmission MOOC course based on the network audio data decoding technology can not only improve students' academic achievement, but also enhance their learning ability, consequently achieving good teaching effects. It has the following advantages: Firstly, compared with the traditional teaching method, MOOC course has a strong degree of freedom, and learners can decide whether to learn or not, as well as the learning time and place. As a result, MOOC can prevent learners from wasting precious time in meaningless learning and apply their time to 
meaningful learning so as to improve the learning results. Secondly, dramatical changes have taken place in the positions of teachers and learners. The learning process is controlled by learners instead of teachers, and learners have higher initiative and freedom degree in the learning process. Most importantly, the subjects of this study are students majoring in journalism. As journalists, they must have excellent critical thinking and discrimination ability, which is formed based on the understanding of all kinds of knowledge and culture. This teaching mode can realize cross-specialty and cross-school cooperation and resource sharing through MOOC platform. Therefore, students majoring in journalism and transmission can receive courses in other colleges and specialties to expand their knowledge.

As a whole, students in experiment group have high recognition of 3D multimedia teaching platform. Most students can accept the new teaching mode and obtain improvement of learning interest and improve their attainment of professional courses. Through 3D multimedia teaching platform, students in architecture specialty have indepth understanding of urban-rural master plan, and they can directly know relevant architecture distribution and characteristics. Meanwhile after class, students and teachers can maintain communication conveniently through $3 \mathrm{D}$ multimedia teaching platform. Students can download relevant materials, propose questions, browse question records, and complete content extension through the system. Teachers can know students' real-time dynamic, adjust teaching objective constantly so as to obtain better teaching effect. 3D multimedia teaching platform is suitable for different stages and different subjects. Using 3D model generated by 3D virtual technology can bring more direct feeling to students and achieve the objective of teaching mode reform and teaching quality improvement.

\section{Conclusions}

In the Internet era, MOOC has become a kind of teaching method with broad development prospects. Various courses are gradually offered to learners in the form of MOOC. The practice results show that the News Transmission MOOC course based on the network audio data decoding technology can not only improve students' academic achievement, but also enhance their learning ability, thus achieving good teaching effects. The following are its advantages:

1. Compared with the traditional teaching method, MOOC course has a strong degree of freedom, and learners can decide whether to learn or not as well as the learning time and place. Therefore, MOOC can prevent learners from wasting precious time in meaningless learning and apply their time to meaningful learning so as to improve the learning results.

2. Under this condition, great changes have taken place in the positions of teachers and learners. The learning process is controlled by learners instead of teachers, and learners have higher initiative and freedom degree in the learning process.

3. Most importantly, the objects in this study are the students majoring in journalism. As journalists, they must have excellent critical thinking and discrimination ability, which is formed based on the understanding of various aspects of knowledge and 
culture. Moreover, this teaching mode can realize cross-specialty and cross-school cooperation and resource sharing through MOOC platform, so that students majoring in journalism and transmission can receive courses in other colleges and other specialties to expand their knowledge.

\section{References}

[1] Huang, Y.H., Yu, Z.H., Xie, C., Shi, D.Y., \& Zhou, X.X. Study on the application of electric power big data technology in power system simulation. Proceedings of the CSEE, 2015, vol. 35(1), pp. 13-22.

[2] Kop, R. The challenges to connectivist learning on open online networks: learning experiences during a massive open online course. International Review of Research in Open \& Distance Learning, 2011, vol. 12(3), pp. 19-38. https://doi.org/10.19173/irrodl.v12i3.882

[3] Fini, A. The technological dimension of a massive open online course: the case of the cck08 course tools. International Review of Research in Open \& Distance Learning, 2009, vol. 10(5), pp. 1-26. https://doi.org/10.19173/irrodl.v10i5.643

[4] Deboer, J., Ho, A.D., Stump, G.S., \& Breslow, L. Changing "course": reconceptualizing educational variables for massive open online courses. Educational Researcher, 2014, vol. 43(2), pp. 74-84. https://doi.org/10.3102/0013189X14523038

[5] Barak, M., Watted, A., \& Haick, H. Motivation to learn in massive open online courses: examining aspects of language and social engagement. Computers \& Education, 2016, vol. 94, pp. 49-60. https://doi.org/10.1016/j.compedu.2015.11.010

[6] Zhuhadar, L., Kruk, S.R., \& Daday, J. Semantically enriched massive open online courses (moocs) platform. Computers in Human Behavior, 2015, vol. 51(PB), pp. 578-593.

[7] Chacón-Beltrán, R. Massive online open courses and language learning: the case for a beginners' English course. Procedia - Social and Behavioral Sciences, 2014, vol. 141, pp. 242-246. https://doi.org/10.1016/j.sbspro.2014.05.042

[8] Liang, W.N., Gan, H.J., \& Min, L. Establishment of curriculum knowledge system of diagnostics of traditional Chinese medicine based on times of massive open online course. Medical Innovation of China, 2017, vol. 26, pp. 133-137.

[9] Wang, S.H., Zhou, Q. Dissimilation and reconstruction of relations between college students and teachers in the era of mooc. Education \& Teaching Research, 2017, vol. 31(2), pp. 58-62.

[10] Root Kustritz, M.V. Canine theriogenology for dog enthusiasts: teaching methodology and outcomes in a massive open online course (mooc). Journal of Veterinary Medical Education, 2014, vol. 41(1), pp. 9-18. https://doi.org/10.3138/jvme.0813-112R1

[11] Yan, J.J. Study on the blending teaching mode of college English based on the background of MOOC. Journal of Anhui Science \& Technology University. 2017, vol. 31(4), pp. 111115.

[12] Koka, A., \& Hagger, M.S. Perceived teaching behaviors and self-determined motivation in physical education: a test of self-determination theory. Res Q Exerc Sport, 2010, vol. 81(1), pp. 74-86. https://doi.org/10.1080/02701367.2010.10599630

[13] Al Mahmud, A. Constructivism and reflectivism as the logical counterparts in tesol: learning theory versus teaching methodology. Teflin Journal, 2013, vol. 24(2), pp. 237-257. 
Paper-MOOC Teaching Mode of News Transmission Based on Network Audio Data Decoding Techno..

\section{$7 \quad$ Author}

Chune SHEN is a Lecturer of College of Literature and Media, Hubei University of Arts and Science, Xiangyang 441053, China. Her research interests include Internet Communication and new media (564842959@qq.com)

Article submitted 17 March 2018. Final acceptance 25 April 2018. Final version published as submitted by the author. 